\title{
Milieux relationnels Xpey' : un cadre analytique pour conceptualiser l'équité en santé autochtone
}

\author{
Alexandra Kent, M.P.H. (1,2); Charlotte Loppie, Ph. D. (1); Jeannine Carriere, Ph. D. (1); \\ Marjorie MacDonald, Ph. D., R.N. (1,2,3); Bernie Pauly, Ph. D., R.N. $(1,2,3)$
}

Cet article a fait l'objet d'une évaluation par les pairs.

\section{Résumé}

Introduction : Si la recherche sur l'équité en santé et la recherche sur la santé autochtone ont toutes deux pour objectif de promouvoir des résultats équitables en santé parmi les populations marginalisées et à moindre accès aux soins, elles fonctionnent souvent de manière indépendante et sans collaboration, conduisant à une sous-représentation des populations autochtones dans la recherche sur l'équité en santé relativement au fardeau disproportionné des inégalités subies. Dans cet article méthodologique, nous présentons Milieux relationnels Xpey', un cadre analytique qui décrit certains obstacles et éléments facilitateurs en matière d'équité en santé pour les peuples autochtones.

Méthodologie : La recherche sur l'équité en santé doit se concentrer sur les populations autochtones et les méthodologies autochtonisées, changement qui pourrait combler les lacunes dans les connaissances et contribuer à combler le fossé observé en santé autochtone. Dans ce cadre, le programme de recherche Optique d'équité en santé publique (Equity Lens in Public Health, ELPH) a adopté le cadre analytique Milieux relationnels Xpey' pour que la recherche sur la priorisation et la mise en œuvre de l'équité en santé soit davantage centrée sur les populations autochtones. Le cadre analytique a ajouté à notre méthodologie une optique autochtonisée d'équité en santé qui a facilité la reconnaissance des déterminants sociaux, structurels et systémiques de la santé autochtone. Pour éprouver ce cadre analytique, nous avons mené une étude de cas pilote portant sur l'une des autorités sanitaires régionales de la Colombie-Britannique, consistant en un examen des politiques et des plans de référence et intégrant des entrevues et des groupes de travail avec du personnel de première ligne, des gestionnaires et des hauts dirigeants.

Conclusion : L'application à ELPH de Milieux relationnels Xpey' illustre l'utilité de ce cadre analytique pour explorer et conceptualiser l'équité en santé autochtone dans le système de santé publique de la Colombie-Britannique. Des applications du cadre analytique devront être intégrées aux méthodes de recherche sur les Autochtones.

Mots-clés : équité en santé, accessibilité des services de santé, santé publique, méthodologie de recherche, populations autochtones

\section{Introduction}

Au Canada comme ailleurs dans le monde, deux domaines en émergence dans la recherche en santé sont en évolution rapide et sont susceptibles de se nourrir l'un l'autre : la recherche sur l'équité en santé et la recherche sur la santé autochtone. La recherche sur l'équité en santé fait enquête sur les disparités en matière d'état de santé ou sur la prestation des soins de santé ${ }^{1}$ et elle intègre aussi l'étude des stratégies, des programmes et des politiques de réduction ou d'élimination des inégalités comme de promotion de l'équité en santé. La recherche sur la santé autochtone est l'étude de la santé et du bien-être des populations autochtones, et elle englobe souvent l'application de méthodes de recherche autochtonisées ou de décolonisation, qui
Points saillants

- Les peuples autochtones du Canada subissent des iniquités dans l'accès aux services de santé et dans les résultats en santé, qui sont évitables et injustes.

- La santé autochtone comporte un certain nombre de déterminants, que ce soit des caractéristiques de protection pour la promotion du bien-être et de la résilience ou des facteurs de risque susceptibles de créer des circonstances défavorables ou nuisibles à la santé.

- L'équité en santé autochtone constitue un enjeu crucial dans le système de santé publique de la Colombie-Britannique et doit constituer une priorité pour les chercheurs, les décideurs et les praticiens.

- Prioriser les populations autochtones dans les politiques, la pratique et la recherche en santé publique peut contribuer à améliorer la santé et le bien-être globaux des peuples autochtones.

insufflent des modes de savoir et des protocoles culturels autochtones dans la pratique de la recherche ${ }^{2,3}$.

Ces deux domaines de recherche, alors qu'ils ont pour objectif commun de promouvoir des résultats en santé équitables au sein des populations marginalisées et à moindre accès aux soins, fonctionnent souvent de manière indépendante et sans collaboration ${ }^{2}$. Cette déconnexion est susceptible d'avoir des répercussions sur la façon dont l'équité en santé autochtone est étudiée dans la mesure où elle l'est ${ }^{1}$. C'est pour

Rattachement des auteures :

1. Université de Victoria, Victoria (Colombie-Britannique), Canada

2. Programme de recherche Optique d'équité en santé publique, Victoria (Colombie-Britannique), Canada

3. Centre for Addictions Research of British-Columbia, Victoria (Colombie-Britannique), Canada

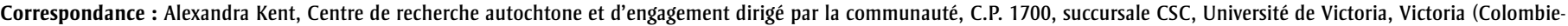
Britannique) V8W 2Y2; tél. : 604-316-2286; courriel : alexandrakent@hotmail.com 
cela que la recherche sur l'équité en santé doit cibler les populations autochtones et utiliser des méthodologies autochtonisées : ce changement majeur pourrait à la fois combler les lacunes dans les connaissances et contribuer également à " combler le fossé » observé en santé autochtone.

Dans cet article, nous présentons Milieux relationnels Xpey', un cadre analytique conçu pour conceptualiser les contextes physiques, interpersonnels et institutionnels où l'équité en santé autochtone peut ou ne peut pas - se réaliser. Nous décrivons l'application de ce cadre analytique au programme de recherche Optique d'équité en santé publique (ELPH) afin d'illustrer sa pertinence pour l'analyse de l'équité en santé autochtone en contexte de santé publique. Milieux relationnels Xpey' a été utilisé par l'équipe de recherche ELPH pour explorer les stratégies utilisées par le système de santé publique de la ColombieBritannique pour réduire les inégalités et améliorer l'équité en santé des peuples autochtones, soit les peuples des Premières Nations, les Inuits et les Métis. Les questions de recherche spécifiques des chercheurs du programme ELPH sont : 1) Quels sont les obstacles et les éléments facilitateurs en matière d'équité en santé dans le système de santé publique de la ColombieBritannique?, 2) Quelles stratégies sont spécifiquement proposées et mises en œuvre par les autorités sanitaires pour intervenir en matière d'équité en santé?, 3) Dans quelle mesure l'équité en santé a-telle été reconnue et priorisée par les autorités sanitaires si l'on se fie aux documents et plans de référence? et 4) Quelles sont les influences contextuelles sur l'établissement des priorités et sur les buts en matière d'équité aux niveaux supérieurs des organismes et du système? Milieux relationnels Xpey' offre un point de vue original sur les peuples autochtones pour répondre à ces questions ainsi qu'une optique autochtone sur l'analyse des données.

\section{Où nous nous situons dans cette recherche}

\section{Alexandra Kent}

Je me situe dans cette recherche comme faisant partie d'une cinquième génération de Canadiens colonisateurs par ascendance britannique et hollandaise. Même si je ne trouve pas important de m'étiqueter en tant que telle, je me situe socialement comme une femme blanche, hétérosexuelle, d'éducation occidentale, anglophone et sans handicap. Je suis consciente que cette identité intersectionnelle me donne les privilèges de plusieurs groupes dominants. De plus, en tant que colonisatrice qui habite sur des territoires non cédés des peuples Songhees, d'Esquimalt et WֵSÁNEĆ, je suis impliquée dans l'histoire coloniale canadienne comme bénéficiaire de la dépossession et de la soumission des peuples autochtones. Je participe à cette recherche avec humilité et en comprenant que je ne représente ni les peuples autochtones, ni leur savoir, ni leur vision du monde, ni leurs optiques culturelles. J'espère contribuer au programme de décolonisation en tant qu'alliée non autochtone par un engagement critique dans une recherche en santé publique. J'ai eu le privilège de travailler comme attachée de recherche et, plus récemment, comme adjointe à la recherche pour le programme de recherche Optique d'équité en santé publique (ELPH) et pour le Centre de recherche autochtone et d'engagement dirigé par la communauté (CIRCLE), qui ont formé un partenariat pour offrir une dimension et des connaissances autochtones au projet ELPH. Cette expérience à ELPH et au CIRCLE m'a apporté une perspective originale qui intègre l'enseignement occidental et les modes de savoir autochtones, un peu comme un « double regard $»^{4}$. C'est de cette position et avec une conscience critique de ma situation que je présente Milieux relationnels Xpey’ avec mes collègues.

\section{Charlotte Loppie}

Je suis d'ascendance Mi'kmaq et acadienne française de Nouvelle-Écosse. J'ai le plaisir d'être visiteuse du territoire des peuples Songhees, d'Esquimalt et ẂSÁNEĆ depuis 2009. Je réponds aux besoins de recherche en santé des communautés et collectivités autochtones depuis 1995 et je donne des cours sur la santé autochtone à l'École de santé publique et de politique sociale de l'Université de Victoria, où je suis également directrice du Centre de recherche autochtone et d'engagement dirigé par la communauté (CIRCLE). Je ne suis pas chercheure pour le projet ELPH, mais l'équipe ELPH m'a demandé de participer à l'élaboration de Milieux relationnels Xpey', un cadre qui, je l'espère, soutiendra l'équité en santé autochtone en ColombieBritannique et ailleurs.

\section{Jeannine Carriere}

Mon nom cri est Sohki Aski Esquao, qui signifie femme de la Terre puissante. Mon nom anglais est Jeannine Carriere. Je suis une femme métisse originaire de la région de la rivière Rouge, au Manitoba, et je vis sur le territoire des peuples Cowichan, sur l'île de Vancouver, depuis 2005. J'enseigne sur le territoire des Songhees, Esquimalt et WSÁNEĆ, depuis 1994 à l'Université de Victoria et depuis 2005 à l'École de travail social. Mes domaines de recherche sont l'adoption et l'identité pour les enfants autochtones, les méthodologies de recherche autochtones, le bien-être des enfants et des familles métis et les relations familiales en contexte de travail du sexe. Grâce à ces champs d'intérêt, j'ai eu la chance de publier ainsi que de créer des réseaux avec des personnes formidables, comme celles qui participent au projet ELPH. J'en suis reconnaissante.

\section{Marjorie MacDonald}

Je participe à cette recherche en tant que femme blanche, hétérosexuelle, scolarisée, sans handicap et de la classe moyenne. Cela m'a apporté des privilèges qui ne sont pas accessibles à beaucoup d'autres dans la société. Même si mon arrière-grand-mère maternelle était crie, ses descendants ont tous été élevés comme des colonisateurs blancs, malheureusement sans connaître ni reconnaître leur héritage cri. Ainsi, nous sommes tous impliqué dans la colonisation et l'oppression des peuples autochtones de ce pays, même des membres de notre propre parenté. J'ai vécu et travaillé dans la réserve Blackfoot, à Gleichen (Alberta), où j'ai appris à connaître et à aimer les traditions et la vision du monde des PiedsNoirs. Cette expérience m'a conduite à consacrer ma carrière à la santé publique, à ses valeurs communautaires et à son éthique collectiviste. Cependant, je ne me réclame pas pour autant d'un savoir, d'une situation ou d'un privilège particuliers dans cette recherche relativement à cette expérience. En tant qu'alliée engagée, je reconnais avec gratitude et humilité mon statut de visiteuse dans ces territoires non cédés des peuples Songhees, Esquimalt et WSÁNEĆ.

\section{Bernadette Pauly}

J'appartiens à la troisième génération de colonisateurs d'ascendance allemande. Ma situation sociale est celle d'une femme blanche, hétérosexuelle, sans handicap, de classe moyenne et scolarisée. J'ai bénéficié de divers opportunités et privilèges en raison de ma position sociale. Je suis colonisatrice et je vis et travaille sur les territoires non cédés des peuples Songhees, Esquimalt et WSÁNEĆ depuis 2000. À ce titre, je suis impliquée dans l'histoire 
coloniale du Canada et dans l'action de nombreux colons canadiens, qui ont conduit au déplacement et à l'oppression de nos peuples autochtones. Je n'ai pas d'appartenance autochtone et je ne possède pas de connaissance intime des savoirs, de la vision du monde ou de l'optique culturelle des Autochtones : mon but dans cette recherche est plutôt d'adopter une approche respectueuse et humble dans l'espoir qu'une mobilisation critique contribuera au programme de décolonisation. Je suis extrêmement reconnaissante de cette occasion de travailler avec d'éminentes chercheures autochtones pour ce travail et pour d'autres études, et j'ai appris énormément de choses tout au long de ces processus. Je vous en remercie.

\section{Le programme de recherche Optique d'équité en santé publique (ELPH)}

Le programme de recherche Optique d'équité en santé publique (ELPH) est un programme sur cinq ans financé par l'IRSC en 2011 et issu de la Core Public Health Functions Research Initiative (CPHFRI) lancée en 2005. La CPHFRI et ELPH appartiennent tous deux spécifiquement au système de santé publique de la ColombieBritannique et fonctionnent en partenariat étroit avec, entre autres, les cinq autorités sanitaires régionales de la province, le ministère de la Santé et l'Autorité provinciale des services de santé. Ces partenariats étaient déjà solides avant la création d'ELPH en 2011. L'Autorité sanitaire des Premières Nations (ASPN), invitée quant à elle à former un partenariat en 2013 (année de sa création officielle) avec ELPH, a décliné cette participation, décision que nous respectons et que nous intégrons comme une limite à cette étude.

Le programme de recherche ELPH est consacré à l'élaboration et à l'application d'une optique d'équité en santé publique et à ses implications pour la réduction des inégalités en santé5. Il porte en particulier sur la promotion de la santé mentale, sur la prévention des maladies mentales et sur les effets néfastes de l'alcool et des drogues. Il est divisé en quatre études, à la fois distinctes et interreliées. On a utilisé Milieux relationnels Xpey' pour une étude de cas dans le cadre de l'étude 1 d'ELPH, Priorités et stratégies en matière d'équité en santé, dont le but est de mener des études de cas individuelles au sein des cinq autorités sanitaires régionales afin de déterminer : 1) quelles sont les activités en cours sur l'équité en santé et la réduction des inégalités, 2) si et comment les problèmes d'équité en santé ont été priorisés, 3) quelles sont les influences contextuelles sur les priorités et les plans et stratégies d'équité en santé et 4) ce qui explique ces changements au cours de l'étude et comment on les explique. Chaque autorité sanitaire constitue un cas individuel permettant d'enregistrer les influences contextuelles à l'échelle régionale et de faciliter les comparaisons entre cas. Le modèle par étude de cas permet aussi l'analyse comparative dans le temps (référence et suivi) pour évaluer les changements dans l'adoption et la mise en œuvre de l'équité en santé en tant que priorité.

Le projet ELPH utilise l'analyse situationnelle, une approche de recherche qui recourt à une méthodologie de théorisation post-moderne ancrée pour connaître et décrire les mondes sociaux et les sphères d'action permettant de comprendre les éléments humains et non humains, les interactions et le contexte d'une situation donnée $^{6}$. Conformément à la méthodologie de Clarke ${ }^{6}$, l'analyse situationnelle est utilisée pour faciliter l'accès aux données et l'analyse de multiples connexions et relations susceptibles d'avoir une influence sur les diverses activités. Un monde ordonné, relationnel et social ainsi que des cartes de positionnement permettent visuellement de comprendre les phénomènes étudiés et la complexité inhérente à une situation. Pour faire connaître les résultats de l'étude ELPH, nous allons produire des rapports de cas régionaux de même qu'une analyse globale à l'échelle de la province résumant les conclusions relatives à l'application et à la mise en œuvre de l'équité en santé au sein des autorités sanitaires ${ }^{5}$. Un rapport de l'analyse situationnelle sera également diffusé.

\section{Contexte}

Dans la proposition d'origine d'ELPH, on présentait les inégalités en santé subies par les peuples autochtones (à savoir les membres des Premières Nations, les Inuits et les Métis) comme un problème spécifique à la Colombie-Britannique, au même titre que les inégalités en santé subies par d'autres sous-populations. L'équipe ELPH s'est aperçue qu'il était nécessaire de porter une attention particulière à l'équité en santé pour les peuples autochtones, ce qui nécessitait un cadre analytique culturellement plus pertinent. C'est ainsi que les chercheurs principaux se sont adressé aux chercheures autochtones Charlotte Loppie (CL, auparavant Reading) et Jeannine Carriere (JC), qui ont mis au point le cadre analytique Milieux relationnels Xpey' pour servir de base à une analyse parallèle utilisant une optique autochtone afin d'explorer le rôle de la santé publique dans l'équité en santé autochtone.

\section{Le modèle Haudenosaunee Gusweñta (ou Two Row Wampum)}

L'équipe ELPH a choisi le Haudenosaunee Gusweñta, ou Two Row Wampum, comme modèle pour conceptualiser la relation entre l'utilisation de l'analyse situationnelle par ELPH et l'approche de Milieux relationnels Xpey'. Le Gusweñta, ou Two Row Wampum, est une ceinture perlée qui a été échangée à la signature du Traité de Niagara en 1764. La ceinture illustre deux embarcations (un canot des Premières Nations et un navire européen) qui naviguent côte à côte sur une rivière, et aucun n'essaie de diriger l'autre ni de croiser son trajet, ce qui symbolise le respect mutuel et l'absence d'interférence? ${ }^{7}$. À la manière de Two Row Wampum, notre analyse est constituée de processus parallèles qui ont en commun un objectif général et un ensemble de données. L'approche autochtone et l'approche occidentale sont considérées comme à la fois distinctes et d'une importance égale, et nous utilisons leurs forces respectives pour permettre "une vision plus large, plus profonde et plus féconde que celle qu'apporterait chaque perspective si elles étaient en permanence isolées $»^{4}$ [traduction]. De plus, nous évitons de fusionner les deux systèmes de connaissance ou d'essayer d'intégrer de force le savoir autochtone à un paradigme occidental.

\section{État des connaissances}

La recherche d'ELPH est ancrée à un corpus de littérature sur l'équité en santé et les systèmes de santé publique. Même si les connaissances et la sensibilisation augmentent dans ces domaines, relativement peu de recherches les ont rapprochés l'un de l'autre ou les ont reliés à la santé autochtone. En 2010, la Coalition canadienne pour la recherche en santé mondiale s'est associée au Centre de recherche sur la santé des Autochtones (devenu le Centre de recherche autochtone et d'engagement dirigé par la communauté [CIRCLE]) pour un projet intitulé " Linking Equity Methods Research with Global Indigenous 
Health Research » (Relier la recherche sur les méthodes d'équité à la recherche sur la santé autochtone mondiale). Ce projet était constitué d'une analyse du milieu, d'un inventaire des outils et d'un atelier pour l'élaboration d'un plan de travail destiné à produire un programme de collaboration entre les deux domaines de recherche ${ }^{2,8}$. Les chercheurs ont conclu : "Même si la recherche sur les méthodes d'équité a progressé au cours de la dernière décennie, les travaux doivent être poursuivis sur des données de recherche axées sur la santé des populations autochtones $»^{8, p .2}$ [traduction]. Les sections suivantes présentent divers éléments d'information pertinents pour décrire le contexte de notre recherche.

\section{Équité en santé}

On parle habituellement dans les milieux scientifiques, pour définir l'inégalité en santé, de « différences en santé qui, en plus d'être inutiles et évitables, sont aussi considérées comme inéquitables et injustes $»^{9, p .5}$ [traduction]. À l'opposé, on peut parler d'équité lorsque les membres marginalisés de la société ont accès aux normes de santé les plus élevées, c'est-à-dire celles de l'état de santé des personnes les plus avantagées $^{10}$. En Colombie-Britannique comme ailleurs, les peuples autochtones subissent un fardeau d'inégalités disproportionné. Il n'existe pas de définition officielle et universellement reconnue des " peuples autochtones ", car chaque communauté, nation ou collectivité a le droit de se définir et de se nommer comme elle l'entend. De façon générale, on peut entendre par peuples, communautés et nations autochtones ceux qui ont « une continuité historique avec les sociétés qui se sont développées sur leurs territoires avant leur conquête et la colonisation [et qui] se considèrent euxmêmes comme distincts des autres secteurs des sociétés prédominantes aujourd'hui sur ces territoires ou sur des parties de ceuxci $»^{2}$ [traduction].

De nombreux chercheurs ont examiné l'état de santé des populations autochtones au Canada et ailleurs dans le monde et ils ont établi des liens entre cet enjeu et des inégalités en matière de déterminants de la santé qui vont au-delà des comportements personnels et des caractéristiques génétiques pour englober des facteurs sociopolitiques plus larges qui, de différente façons, ont une profonde influence sur la santé $e^{11-16}$. Il existe un mouvement récent en recherche sur la santé autochtone qui s'éloigne de l'optique pathologisante et sensationnaliste des disparités ou des "déficiences » perçues dans les communautés autochtones pour se centrer plutôt sur le rôle des injustices structurelles dans le façonnement des conditions sociales.

ELPH ne se donne pas pour objectif de reproduire les résultats d'autres études qui ont fait ressortir les états de santé inéquitables : cette recherche vise plutôt à analyser le rôle des systèmes et des structures de santé publique dans la perpétuation ou l'arrêt de ces inégalités. Les systèmes de soins de santé, en particulier le système de santé publique, ont pour mandat de fournir des services qui favorisent, restaurent ou maintiennent la santé de la population. Le Cadre d'orientation en matière de santé publique de la Colombie-Britannique ${ }^{17}$ rappelle les fonctions de base de la santé publique, notamment prévenir les maladies et les blessures, protéger les populations contre les risques pour la santé et promouvoir des politiques, un environnement et des comportements publics sains. La santé publique a deux objectifs d'ordre moral fondamentaux : promouvoir la santé de la population et réduire les inégalités en santé $^{18}$. C'est pourquoi le système de santé publique a été reconnu comme un lieu d'intervention important pour promouvoir l'équité en santé et réduire les inégalités. Cependant, en tant que système colonial, il risque aussi de contribuer à augmenter les inégalités, en particulier en matière de difficultés d'accès aux soins de santé pour les peuples autochtones.

\section{Analyse du contexte historique et institutionnel}

Les difficultés d'accès à l'équité en santé pour les peuples autochtones risquent souvent de se manifester dans le système de santé publique sous forme de gouvernance fragmentée, de complexité des compétences territoriales, de lacunes dans la gamme de services et de manque de reddition de comptes gouvernementale. Ces questions ont été relevées et ont fait l'objet de problématisation dans divers rapports, dont le rapport final de la Commission de vérité et réconciliation du Canada ${ }^{16}$, le rapport final de la Commission royale sur les peuples autochtones ${ }^{19}$ et le Rapport de l'administrateur en chef de la santé publique ${ }^{13}$. Le système de gouvernance de la santé autochtone du Canada a été qualifié de " dédale bureaucratique » ${ }^{11, p .5}$ [traduction] et de « "mosaïque" de mesures législatives, de politiques et de relations ${ }^{20, p .1}$. Ces problèmes trouvent leur origine dans la Loi de 1867 sur l'Amérique du Nord britannique (Loi constitutionnelle de 1867), qui stipule au paragraphe 91(24) que " les Indiens et les terres qui leur sont réservées " sont de la compétence du gouvernement fédéral et que les soins de santé, les services sociaux et l'éducation sont de compétence provinciale $^{21}$.

Les limites de compétences décrites dans la Loi constitutionnelle de 1867 peuvent sembler claires en théorie, mais elles se sont révélées ambiguës et trop compliquées en pratique. Aux divisions qui existent entre paliers de gouvernement se sont ajoutées d'autres divisions, selon les ancêtres, les lieux de résidence et les ententes sur les revendications territoriales. Ces multiples divisions dans les compétences provoquent de la confusion lorsqu'il s'agit de fournir des services de santé aux populations autochtones et elles conduisent à un chevauchement des responsabilités entre les autorités des gouvernements fédéral, provinciaux et territoriaux. Au fil des années, les peuples autochtones ont tenté d'avoir un meilleur contrôle sur les décisions relatives aux politiques, aux programmes et aux services de santé, mais il est difficile de savoir si cela a réduit ou aggravé les conflits de compétences ${ }^{20,22-24}$.

\section{Le système de santé publique de la Colombie-Britannique}

Le BC Core Public Health Functions Framework $^{25}$ puis le Guiding Framework for Public Health ${ }^{17}$ qui a pris sa suite soulignent que les fonctions de base de la santé publique sont sous la responsabilité de l'ensemble du système de santé, des organismes non gouvernementaux et privés ainsi que de la société civile. C'est dire que les fonctions de santé publique ne sont pas uniquement exercées par le système de santé publique officiel et les praticiens de la santé publique traditionnels. Cependant, dans le programme de recherche d'ELPH, nous nous concentrons sur le système de santé publique officiel, dont les politiques et les pratiques sont axées sur la promotion de la santé, la prévention de la maladie et des blessures, la protection ainsi que la surveillance et l'évaluation de la santé, et non sur le traitement, qui relève de l'ensemble du système de soins de santé.

Le système de santé publique officiel de la Colombie-Britannique est composé du 
ministère de la Santé, de l'Autorité provinciale des services de santé, de cinq autorités sanitaires régionales (Northern Health, Interior Health, Fraser Health, Vancouver Coastal Health, Vancouver Island Health Authority) et de l'Autorité sanitaire des Premières Nations $(\mathrm{ASPN})^{26}$. En décembre 2001, le gouvernement provincial a fusionné les 52 autorités sanitaires en cinq autorités régionales dans le but de simplifier un système compliqué et coûteux ${ }^{26}$. Les autorités sanitaires régionales sont responsables de la planification, de la gestion et de la prestation des programmes et des services de santé dans leur zone géographique $^{26}$. Le ministère de la Santé appuie et finance les programmes et les services de toutes les autorités sanitaires et fournit des lignes directrices pour assurer un niveau de qualité normalisé à l'ensemble des zones géographiques et des populations ${ }^{26}$. L'Autorité provinciale des services de santé collabore également avec les autorités sanitaires régionales et les organismes qui les appuient pour planifier et coordonner des programmes provinciaux et des services de santé spécialisés à l'échelle de la province $^{26}$. Ces autorités gouvernementales collaborent pour fournir des services de santé complets à tous les BritannoColombiens.

L'ASPN, qui est l'autorité sanitaire la plus récente en Colombie-Britannique, représente et dessert spécifiquement les Premières Nations. Elle fait partie d'un cadre de gouvernance de la santé rassemblant les Premières Nations, la province de la Colombie-Britannique et le gouvernement du Canada, et il s'agit du premier du genre au Canada. Ce cadre tripartite facilite le transfert de responsabilités à l'ASPN pour la planification, la conception, la gestion et la prestation des programmes et des services de santé des Premières Nations de la Colombie-Britannique depuis la Direction générale de la santé des Premières Nations et des Inuits de Santé Canada. Si ce transfert a eu lieu officiellement le $1^{\text {er }}$ octobre 2013, il est l'aboutissement de consultations et de négociations intensives antérieures, consacrées par trois ententes sur la santé : le Transformative Change Accord: First Nations Health Plan (2006), le Plan tripartite pour la santé des Premières nations (2007) et l'Accord-cadre tripartite de la Colombie-Britannique sur la gouvernance de la santé des Premières Nations $(2011)^{27}$. Ces trois ententes forment un cadre juridiquement contraignant qui décrit la structure et le mandat de gouvernance de la santé des Premières Nations, les engagements de financement du gouvernement fédéral et des provinces de même qu'une vision unifiée pour un système de services de santé intégré.

La création de l'ASPN n'augmente pas la complexité de compétences liées à des systèmes de services de santé distincts pour les Premières Nations et le reste de la population : au contraire, elle renforce les liens entre l'ASPN, Santé Canada, le ministère de la Santé de la Colombie-Britannique et les autorités sanitaires de la ColombieBritannique $^{27}$. La coordination et la collaboration entre ces partenaires devraient à long terme améliorer la qualité, l'accessibilité et l'efficacité des programmes et des services de santé pour les Premières Nations en réduisant leur complexité et en favorisant des prestations de services plus intégrées. Ce nouveau cadre améliore également le contrôle des Premières Nations sur la gouvernance de la santé et renforce l'acceptabilité de ces services par l'intégration de modèles de bien-être culturellement pertinents. L'ASPN a un mandat « en action pour la collectivité, à l'échelle de la nation » qui représente la diversité des peuples et des cultures des Premières Nations de la Colombie-Britannique ${ }^{22}$. Cependant, le mandat de l'ASPN s'applique exclusivement aux peuples des Premières Nations inscrits et ne répond donc pas aux besoins des autres groupes autochtones (les Métis, les Inuits, ceux qui ne sont pas inscrits) de la Colombie-Britannique ${ }^{20}$. Le gouvernement fédéral fait en effet la distinction entre Indiens inscrits et Indiens non inscrits, un Indien inscrit étant une personne inscrite conformément à la Loi sur les Indiens ${ }^{20}$. On prévoit qu'avec le temps, l'ASPN jouera un rôle plus important et exercera une influence accrue sur la planification des services et leur prestation à une population autochtone plus large en Colombie-Britannique, et potentiellement aussi à la population non autochtone ${ }^{27}$.

\section{Méthodologie et résultats}

\section{Élaboration du cadre analytique}

En 2014, CL et JC ont reçu des chercheurs principaux le mandat d'élaborer un cadre analytique pour guider l'analyse d'une étude de cas d'équité en santé autochtone pour l'étude 1 d'ELPH. Le cadre de l'Organisation mondiale de la santé (OMS) pour les déterminants sociaux de la santée ainsi qu'une théorie autochtone critique ${ }^{29}$ ont servi à documenter l'élaboration de ce cadre analytique. En combinant les concepts de déterminants proximaux, intermédiaires et distaux avec ceux associés à l'oppression coloniale, CL et JC ont façonné un cadre spécifiquement centré sur les milieux relationnels, systémiques et structurels au sein desquels la santé publique autochtone prend forme. Ce cadre est conforme aux perspectives théoriques de l'ensemble du programme de recherche $\mathrm{ELPH}^{5}$, en particulier l'analyse intersectionnelle, qui est centrée sur différents lieux sociaux, forces et structures de pouvoir qui modèlent la vie humaine $e^{30,31}$.

Alexandra Kent (AK) a appliqué ce cadre aux données provenant de l'une des autorités sanitaires de la Colombie-Britannique. Les détails de l'étude de ce cas pilote (dont le choix de l'autorité sanitaire) n'ont pas encore été divulgués, que ce soit aux utilisateurs de connaissances ou au public. Ce cadre analytique a ensuite été appelé Milieux relationnels Xpey', après consultation avec Shauna Underwood, une conseillère autochtone de l'Université de Victoria. Xpey' signifie cèdre rouge de l'Ouest en Hul'q'umi'num', un dialecte de la famille linguistique Halkomelem parlé principalement par les Premières Nations de l'île de Vancouver. Il n'existe aucun mot unique pour désigner un arbre en Hul'q'umi'num', ce pourquoi nous avons choisi le cèdre rouge de l'Ouest, pour sa portée culturelle en tant que remède sacré.

L'expression Milieux relationnels Xpey' relève d'une métaphore de l'arbre créée par CL pour rendre compte des déterminants proximaux, intermédiaires et distaux de la santé autochtone ${ }^{15}$. Cette approche métaphorique issue de la Terre utilise le biomimétisme pour fournir une analyse plus profonde de concepts abstraits fondée sur une compréhension du monde naturel ${ }^{32}$. Quoique non spécifique des cultures autochtones, la métaphore de l'arbre rend compte d'une sensibilité culturelle autochtone. CL l'explique ainsi [traduction] ${ }^{33, p .4}$ :

Nous sommes habitués à concevoir un arbre sous la forme de trois éléments interconnectés : la cime (les feuilles et les branches), le tronc et les racines. Chaque partie de l'arbre dépend non seulement des autres parties pour sa subsistance et son soutien, mais aussi du milieu qui le nourrit et, parfois, l'endommage. 
Alors que la santé des racines, invisibles sous la terre, a une forte influence sur la santé de l'arbre, l'état de la cime constitue souvent un indicateur de la santé globale de l'arbre. Milieux relationnels Xpey' applique cette sagesse aux contextes physiques et théoriques, ou " milieux relationnels », dans lesquels l'équité en santé se manifeste au sein des systèmes et des structures de la santé publique.

Dans Xpey', les milieux relationnels sont conçus comme les trois composantes d'un arbre : la cime, le tronc (ou centre) et les racines (figure 1). Comme pour un arbre, les milieux de la cime influencent la santé de l'individu et de la communauté par les liens les plus évidents et directs ${ }^{33}$ : les relations interpersonnelles comme celles entre les prestataires de services et leurs clients ou patients, l'environnement naturel et bâti incluant les obstacles aux services et aux ressources et enfin le positionnement ou la représentation symboliques des identités et des cultures intersectionnelles des personnes.

Comme le tronc d'un arbre, les milieux du centre connectent les milieux de la cime et ceux des racines par des liens qui peuvent soit favoriser la santé soit lui être nuisibles $^{33}$. Ces milieux relationnels ont une influence moins directe sur la santé des individus en tant que telle, mais ils ont une forte influence sur les types de relations et les contextes des milieux de la cime. Ce sont les systèmes d'autorités, politiques et de bureaucratie, les directions et gestions des établissements et des organismes concernés et enfin les systèmes et structures locaux à l'échelle de la communauté.

Enfin, les milieux des racines représentent les contextes historiques, politiques, sociaux et culturels à partir desquels tous les autres milieux relationnels évoluent ${ }^{15}$. CL explique que : "De même que la maladie des feuilles n'est pas la cause de la maladie de l'arbre, les inégalités en santé humaine résultent souvent de la corruption ou de déficiences du système des racines, aussi primordial qu'invisible. » ${ }^{33, p .5}$ [traduction]. Pour la santé autochtone et la gouvernance en santé, ces racines représentent les histoires coloniales et les traumatismes intergénérationnels, les relations et les tractations politiques, les inégalités sociales et matérielles ainsi que les liens culturels effectifs ou rompus. Au sein de ces milieux relationnels de la cime, du tronc et des racines, on trouve des caractéristiques de protection pour la promotion du bien-être et de la résilience aussi bien que des facteurs de risque susceptibles de créer des

FIGURE 1

Représentation visuelle du cadre analytique Milieux relationnels Xpey’

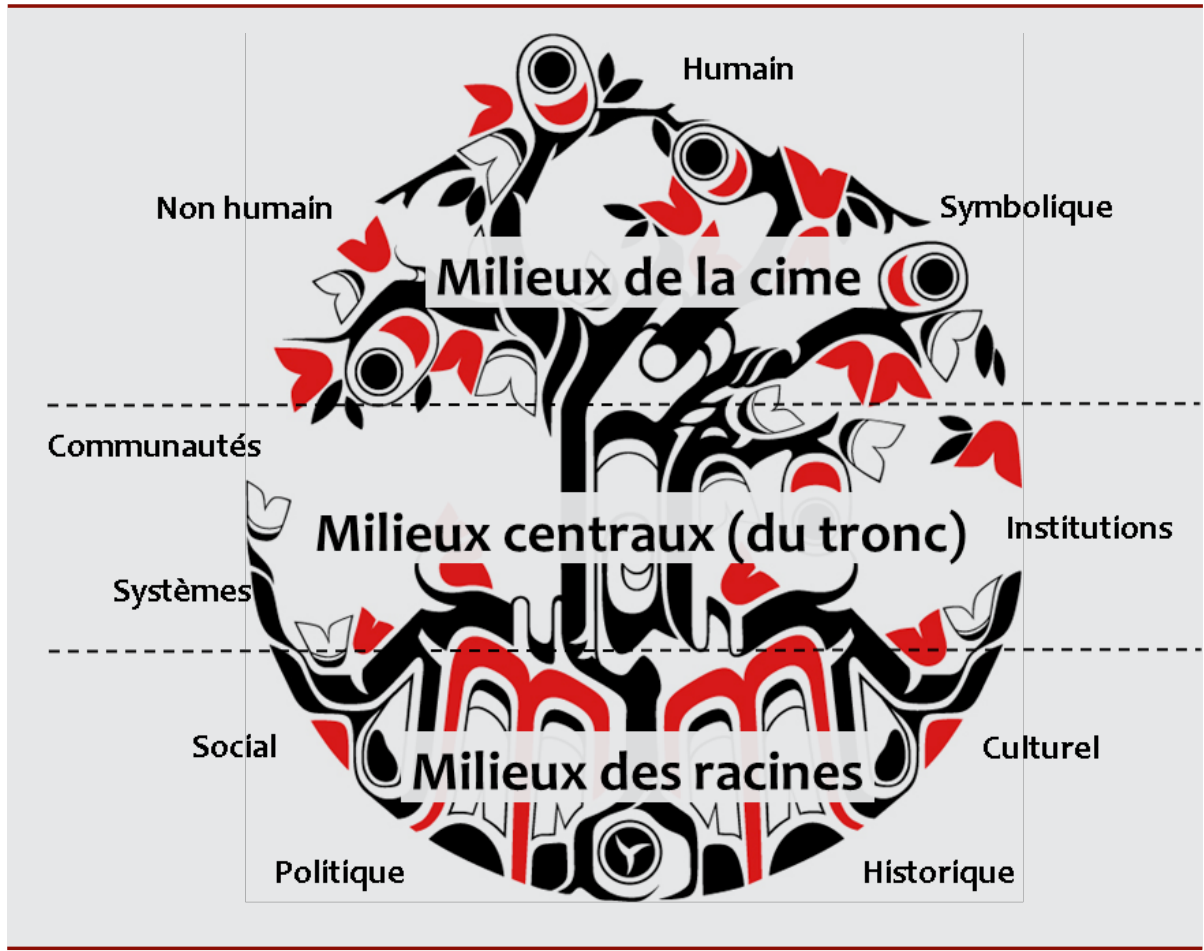

Source : Les auteures ont obtenu l'autorisation de l'artiste, kireihiryu, d'utiliser cette illustration de l'arbre. circonstances défavorables ou de nuire à la santé.

\section{Application du cadre analytique}

$\mathrm{Au}$ cours des quatre dernières années, l'équipe ELPH a rassemblé les plans stratégiques, les plans de service, les plans d'équité en santé, les rapports gouvernementaux et les autres documents pertinents de chacune des autorités sanitaires. La collecte s'est déroulée parallèlement à des entrevues en profondeur et à des groupes de discussion avec du personnel de première ligne, des superviseurs, des gestionnaires et des hauts dirigeants. Les données de référence ont été recueillies en 2012-2013 et des données de suivi de l'été 2015 au printemps 2016.

Toutes les données de l'étude de cas pilote choisies pour être analysées avec le cadre Milieux relationnels Xpey' ont été compilées et codifiées au moyen du logiciel qualitatif NVivo10 version 10 (QSR International Pty Ltd. 2012), qui permet de stocker, d'organiser, de gérer et d'analyser des données qualitatives ${ }^{34}$. Les codes initiaux ont été dérivés de manière déductive en utilisant Milieux relationnels Xpey' afin de les catégoriser plus généralement en milieux de la cime, milieux du centre ou milieux des racines. Une codification inductive a également été utilisée pour enregistrer la profondeur et le détail contextuel du contenu : NVivo10 a permis de créer des étiquettes de code "in vivo " au moyen requêtes de fréquence de mots permettant de connaître les termes et les concepts récurrents dans les sources. Les codes tirés des données servent ainsi de sous-codes au sein des codes globaux issus de la théorie (milieux de la cime, du centre et des racines). Après atteinte du point de saturation, les codes ont été raffinés et triés manuellement en mettant les catégories en relation les unes avec les autres en fonction de leurs similarités conceptuelles jusqu'à obtenir les meilleures affinités. Avec l'intégration de la hiérarchie de codes au cadre théorique, les milieux relationnels étaient nés.

En 2015, CL a obtenu une subvention de planification et de dissémination des IRSC pour tenir une réunion de mobilisation des intervenants avec des représentants du système de santé publique de la ColombieBritannique responsables de la santé autochtone dans leurs organismes respectifs. Début 2016, une réunion d'une journée 
a été tenue avec des dirigeants des services de santé autochtones de chaque organisme du système de santé publique de la Colombie-Britannique, à savoir les cinq autorités sanitaires régionales, l'ASPN, l'Autorité provinciale des services de santé et le ministère de la Santé. La majorité des participants étaient des membres des Premières Nations ou des Métis qui collaboraient étroitement avec des communautés dans la province. Les résultats préliminaires de l'étude de cas pilote ont été présentés aux participants pour qu'ils commentent le cadre analytique Milieux relationnels Xpey'.

Plusieurs réflexions importantes et recommandations clés ont été formulées lors de la réunion de mobilisation des intervenants. Pour tous les participants, l'équité en santé autochtone était une question cruciale au sein du système de santé publique de la Colombie-Britannique et devait constituer une priorité pour les chercheurs. Les intervenants ont aussi grandement apprécié Milieux relationnels Xpey' comme cadre analytique de l'équité en santé autochtone. Cependant, certains participants se sont dits préoccupés par le fait qu'ELPH n’ait pas été conçu à l'origine comme un projet d'équité en santé autochtone, et ont souligné les limites d'une approche ayant consisté à ajouter une dimension autochtone pour une analyse secondaire plutôt que d'avoir orienté la recherche dans une perspective autochtone dès le départ.

\section{Analyse}

ELPH repose sur une théorisation ancrée dans l'analyse intersectionnelle ${ }^{30,31}$ et une théorie de la justice sociale ${ }^{18,35}$, plutôt que sur une théorie post-coloniale critique de la race ou fondée sur des visions du monde autochtones, ce qui constitue une importante limite restreignant l'usage et l'application du cadre analytique Milieux relationnels Xpey' au sein d'ELPH. ELPH est un projet de recherche collaboratif avec les dirigeants de la santé publique des autorités sanitaires de la ColombieBritannique (qui a débuté en 2011, avant que l'ASPN ne soit officiellement intégrée au système de santé publique de la province et à une époque où les ministères autochtones étaient moins présents dans les autorités sanitaires). Une fois l'ASPN établie, les chercheurs en chef d'ELPH ont rencontré des représentants de l'ASPN pour les inviter à être partenaires de la recherche, mais elle avait déjà démarré. Nous avons donc respecté leur décision de ne pas participer et nous avons accepté de les tenir au courant et de leur communiquer les résultats tout au long du processus de recherche. Les données recueillies dans le cadre d'ELPH ne rendent donc pas compte de l'important travail effectué par les peuples autochtones et en collaboration avec eux au sein des autorités sanitaires, en partie à cause du manque de partenariats et du calendrier du projet. Cela constitue une lacune importante dans la représentativité du travail en équité en santé autochtone au sein du système de santé publique de la Colombie-Britannique.

Les travaux de départ et les commentaires générés à la suite de la réunion de mobilisation des intervenants ont été d'une valeur considérable et ont fourni de bons points de repère pour l'application du cadre analytique Milieux relationnels Xpey' à d'autres travaux de recherche sur l'équité en santé. En particulier, l'utilisation du cadre Milieux relationnels Xpey' comme structure analytique doit être ancrée dans les méthodologies de recherche autochtones et dirigée par les peuples autochtones. De plus, les applications à venir du cadre analytique (ou de versions adaptées) devront inclure une représentation des divers groupes culturels (p. ex. les Métis) et offrir une attention particulière à leurs expériences spécifiques. Avec la participation de détenteurs de savoir traditionnel et de leaders organisationnels autochtones à ce cadre analytique et grâce à l'apport de méthodes de recherche autochtones, Milieux relationnels Xpey' pourrait contribuer à structurer des questions de recherche liées à l'équité autochtone au sein du système de santé publique de la Colombie-Britannique et ailleurs.

\section{Conclusion}

Cette application du cadre analytique Milieux relationnels Xpey' illustre son utilité pour l'exploration et la conceptualisation de l'équité en santé autochtone. Il permet de saisir l'importance cruciale des déterminants de l'équité en santé autochtone au sein du système de santé publique de la Colombie-Britannique, tout en étant utilisable dans d'autres études et d'autres contextes. Parmi les applications à venir, il nous semble tout à fait adapté à une analyse ciblée des stratégies de l'ASPN pour la réduction des inégalités et le renforcement de l'équité en santé des peuples autochtones. De plus, Milieux relationnels Xpey’ serait utilisable comme cadre analytique pour explorer le système de santé local d'une communauté autochtone particulière à l'aide d'une recherche-action participative communautaire. Notre expérience d'application de Milieux relationnels Xpey' à une étude de cas pilote dans le cadre d'un programme de recherche d'ELPH nous a permis de conclure que toute application future de ce cadre analytique devra se situer dans un processus de recherche autochtone, nourrie par des protocoles culturels et guidée par des Aînés ou autres détenteurs de savoir traditionnel.

\section{Remerciements}

Les auteures ont reçu le soutien financier des organismes suivants pour la recherche, la rédaction et la publication de cet article : Subvention de fonctionnement des Instituts de recherche en santé du Canada : Subventions programmatiques pour la santé et l'équité en santé (IRSC, subvention $\mathrm{n}^{\circ} 116688$ ) et subvention de planification et de dissémination des Instituts de recherche en santé du Canada : programme d'appui communautaire des instituts (IRSC, subvention $\mathrm{n}^{\circ} 345339$ ).

\section{Conflits d'intérêts}

Les auteures ont déclaré n'avoir aucun conflit d'intérêts potentiel relativement à la recherche, à la rédaction et à la publication de cet article.

\section{Contributions des auteures et avis}

BP et MM ont contribué à la conception de l'étude et de la recherche et à la collecte des données. CL et JC ont fourni des conseils pour mettre à point la méthodologie et ont conçu le cadre analytique. AK a piloté le cadre, interprété les résultats et rédigé le manuscrit, avec les conseils des coauteures. Toutes les auteures ont participé à la révision du manuscrit et ont approuvé la version finale.

Les points de vue exprimés dans cet article sont ceux des auteures et ne doivent pas être considérés comme représentant les opinions des bailleurs de fonds.

Le contenu de cet article et les opinions qui y sont exprimées n'engagent que les auteurs et ne représentent pas forcément les opinions du Gouvernement du Canada. 


\section{Références}

1. Association of American Medical Colleges. The state of health equity research: closing knowledge gaps to address inequities. 2014 [consultation le 13 août 2016]. En ligne à : https:// members.aamc.org/eweb/upload/ The $\% 20$ State $\% 20$ of $\% 20$ Health $\% 20$ Equity \% 20 Research \% 20 - \% 20 Closing \% 20Knowledge \% 20Gaps \% 20 to $\% 20$ Address $\%$ 20Inequities.pdf

2. Centre for Aboriginal Health Research. Linking GIHR and EMR: environmental scan and tool inventory. 2010 [consultation le 18 mai 2016]. En ligne à : https://www.uvic.ca/research/centres /circle/assets/docs/Publications/Enviro \% 20Scan \% 20Linking \% 20GIHR \% 20 and \% 20EMR \% 202.pdf

3. Rigney LI. Internationalization of an Indigenous anticolonial cultural critique of research methodologies: a guide to Indigenist research methodology and its principles. Wicazo Sa Review. 1999;14(2):109-121.

4. Iwama M, Marshall M, Marshall A, Bartlett C. Two-eyed seeing and the language of healing in community based research. Canadian Journal of Native Education. 2009;32(3):3-23.

5. Pauly BM, MacDonald M, Hancock T, Martin W, Perkin K. Reducing health inequities: the contribution of core public health services in BC. BMC Public Health. 2013;13(1): 550. doi: 10.1186/1471-2458-13-550.

6. Clarke AE. Situational Analysis: Grounded Theory after the Postmodern Turn. Thousand Oaks: Sage; 2005.

7. Borrows J. Wampum at Niagara: The Royal Proclamation, Canadian legal history, and self-government. Dans Asch M. (dir.), Aboriginal and treaty rights in Canada: Essays on law, equality, and respect. Vancouver : University of British Columbia Press; 1997. p. 155-172. En ligne à : http://www.sfu.ca/ palys /Borrows-WampumAtNiagara.pdf

8. Canadian Coalition for Global Health Research, Centre for Aboriginal Health Research. Linking equity methods research and global Indigenous health research - Workshop. 2010 [consultation le 7 mai 2017]. En ligne à : http:// www.cgh.uottawa.ca/assets/documents /news_2010_GIHR_equity_release.pdf
9. Whitehead M. The concepts and principles of equity and health. Health Promotion International. 1991;6(3):217228. doi: $10.1093 /$ heapro/6.3.217

10. Sparks M. A health promotion approach to addressing health equity. Global Health Promotion. 2010;17(1): 77-82.

11. Adelson N. The embodiment of inequity: Health disparities in Aboriginal Canada. Can J Public Health. 2005; 96(S2):S45-S61.

12. British Columbia Office of the Provincial Health Officer. Highlights: pathways to health and healing: 2nd report on the health and well-being of Aboriginal people in British Columbia. Provincial Health Officer's Annual Report, 2007. 2007 [consultation le 13 août 2016]. En ligne à : http://www2 .gov.bc.ca/assets/gov/government /ministries-organizations/ministries /health/aboriginal-health-directorate /abohlth11-var7.pdf

13. Butler-Jones D. Notre population et notre santé en chiffres. Dans L'administrateur en chef de la santé publique : Rapport sur l'état de la santé publique au Canada. Ottawa (Ont.) : Agence de la santé publique du Canada; 2008 [consultation le 13 août 2016]. p. 19-33. En ligne à : https://www.canada.ca/fr/sante -publique/organisation/publications /rapports-etat-sante-publique-canada -administrateur-chef-sante-publique /rapport-administrateur-chef-sante -publique-etat-sante-publique-canada -2008.html

14. Health Officers Council of British Columbia. Health inequities in British Columbia: A discussion paper. Public Health Association of British Columbia; 2008.

15. Reading C, Wien F. Health inequalities, social determinants and life course health issues among First Nations people in Canada. National Collaborating Centre for Aboriginal Health. 2009 [consultation le 30 novembre 2016]. En ligne à : https://www.scribd .com/document/189362540/Health -Inequalities-and-Social-Determinantsof-Aboriginal-Peoples-Health-Charlotte -Loppie-Reading-Fred-Wien
16. Commission de vérité et réconciliation du Canada. Honorer la vérité, réconcilier pour l'avenir : sommaire du rapport final de la Commission de vérité et réconciliation du Canada. Montréal : McGill-Queen's University Press; 2015 [consultation le 11 mai 2017]. En ligne à : http://www.trc.ca/websites /trcinstitution/index.php?p $=891$

17. Ministry of Health. Promote, protect, prevent: our health begins here. BC's guiding framework for public health. Province of British Columbia; 2013.

18. Powers M, Faden R. Social Justice: The Moral Foundations of Public Health and Health Policy. New York: Oxford University Press; 2006.

19. Affaires autochtones et Développement $\mathrm{du}$ Nord Canada. Points saillants du rapport de la Commission royale sur les peuples autochtones. Gouvernement du Canada; 1996 [consultation le 13 août 2016]. En ligne à : http:// www.aadnc-aandc.gc.ca/fra/11001000 $14597 / 1100100014637$

20. Lavoie J, Gervais L, Toner J, Bergeron O, Thomas G. À la recherche des Autochtones dans les mesures législatives et les politiques sur la santé, 1970 à 2008 : projet de synthèse politique. Centre de collaboration nationale de la santé autochtone; 2011. En ligne à : https://www.researchgate .net/publication/273729711_Looking _for_Aboriginal_Health_in_Legislation _and_Policies_1970_to_2008_The_ Policy_Synthesis_Project

21. Kelly MD. Toward a new era of policy: health care service delivery to First Nations. International Indigenous Policy Journal. 2011;2(1):1-12.

22. First Nations Health Council. Implementing the vision. BC First Nations health governance. Reimagining First Nations health in BC. First Nations Health Council; 2011.

23. Hirch M. Self-determination in Indigenous health: a comprehensive perspective. Fourth World Journal. 2011;10(2):1-30.

24. Webster P. Local control over Aboriginal health care improves outcome, study indicates. Canadian Medical Association Journal. 2009; 181(11):249-250. doi: 10.1503/cmaj $.109-3072$. 
25. Population Health and Wellness, Ministry of Health Services. A framework for core functions in public health - resource document. Province of British Columbia. 2005 [consultation le 13 août 2016]. En ligne à : http://www.health.gov.bc.ca/library /publications/year/2005/core_ functions.pdf

26. Province of British Columbia. About public health [Internet]. [consultation le 13 août 2016]. En ligne à : http:// www2.gov.bc.ca/gov/content/health /about-bc-s-health-care-system/

27. Gouvernement du Canada. Accordcadre tripartite de la ColombieBritannique sur la gouvernance de la santé des Premières nations. Affaires autochtones et Développement du Nord Canada; 2011.

28. Organisation mondiale de la santé, World Health Organization Commission on Social Determinants of Health. A conceptual framework for analysis and action on the social determinants of health. Genève : OMS; 2010 [consultation le 7 mai 2017]. En ligne à : http://www.who.int/sdhconference /resources/onceptualframeworkfor actiononSDH_eng.pdf

29. Moreton-Robinson A. Introduction: critical Indigenous theory. Cultural Studies Review, 2009;15(2):10-12. doi: 10.5130/csr.v15i2.2034.

30. Hankivsky O, Cormier R. Intersectionality: moving women's health research and policy forward. Vancouver : Women's Health Research Network; 2009.

31. Walby S. Theorizing multiple social systems. Dans : Globalization and inequalities: complexity and contested modernities. London: Sage; 2009. p. 58-100.

32. Wahl DC. Learning from nature and designing as nature: regenerative cultures create conditions conducive to life. [Web Log Post] The Biomimicry Institute [Internet]. 6 septembre 2016 [consultation le 27 septembre 2017]. En ligne à : https://biomimicry.org /learning-nature-designing-nature -regenerative-cultures-create -conditions-conducive-life/
33. Reading C. Structural determinants of Aboriginal peoples' health. Dans : Greenwood M, de Leeuw S, Lindsay NM, Reading C (dir.), Determinants of Indigenous peoples' health in Canada: beyond the social. Toronto (Ont.) : Canadian Scholars' Press; 2015. p. 3-15.

34. QSR International. About NVivo. Technical resource center. [consultation le 13 août 2016]. En ligne à : http://techcenter.qsrinternational.com /desktop/nv10/nv10_about_nvivo_.htm

35. Fraser N. Re-framing justice in a globalizing world. Dans Fraser N, Bourdieu P, Lovel T (dir.), (Mis)recognition, social inequality and social justice. New York: Routledge; 2007. p. 17-35. 